\title{
Modeling Creep Relaxation of Polytetrafluorethylene Gaskets for Finite Element Analysis
}

\author{
Madhu Bharadwaj, Santiago Claramunt, and Sowmianarayanan Srinivasan
}

\begin{abstract}
A typical flange joint consists of one or more gaskets to arrest the leak between the two ends. The important requirements of such gasket is its durability and sealing capacity during its service under operational loads. Many materials qualify for sealing purpose but, Polytetrafluorethylene (PTFE) gaskets have a very high durability and can be used due to its chemical inertness. However, a PTFE gasket will not maintain a long-term seal capability. Once compressed, PTFE gradually relaxes over a period of time to a no load condition, where there is no residual sealing force. This situation results in leakage. Thus there is a need to study the interactions between assembly configuration, initial torque, etc. to relaxation behavior of the gasket as a means to reduce the dwell period (the time between initial torque and re-torque). With an advancement in computational methods it is possible to predict the joint behavior using Finite Element Method (FEM) approach. FEM based study of such complex assembly will be useful only if PTFE gaskets are represented with proper material model. Present paper illustrates a mathematical model, viz., Burger's model to accurately capture the stress relaxation of viscoelastic behavior of a PTFE gasket. The same model is approximated and evaluated in FEM package to determine the predictability of relaxation behavior of PTFE gasket.
\end{abstract}

Index Terms-Burger's model, Polytetrafluorethylene, Prony series, PTFE, stress relaxation, viscoelastic behavior.

\section{INTRODUCTION}

Gaskets are deformable material used between two or more mating parts, intended to prevent the leakage. For the effective working of the gasket the bounce back or retention sealing force in the gasket is very important. Since most of the gaskets are made up of elastomer which are viscoelastic in nature. Viscoelastic material would dissipate the stored energy over the time. This decrease of the stored energy or sealing force is known as stress relaxation. Stress relaxation would lead to leakage over a period of time when it falls below a critical value. Generally stress relaxation studies are done for a short period of time. However, lot of research has been done to extrapolate the stress relaxation behavior based on few accelerated creep relaxation test for long term. Luc Marchand [1] proposed thermo-oxidative disintegration

Manuscript received October 1, 2015; revised January 13, 2016. This work was carried out as part of the activities by Advanced Engineering Group of Infosys Ltd. The authors would like to thanks Infosys Ltd for their financial support and encouragement to publish this paper.

Madhu Bharadwaj is with Infosys Ltd, No. 350, Hebbal Electronics City, Mysore 570 027, India (e-mail: Madhu_Sridharan@infosys.com).

Santiago Claramunt is with Alstom Wind, S.L.U, Roc Boronat, 78, 08005 Barcelona, Spain (e-mail: santiago.claramunt-estecha@power.alstom.com).

Sowmianarayanan Srinivasan is with Infosys Ltd, Electronics City, Hosur Road Bangalore $\quad 560 \quad 100, \quad$ India (e-mail:Sowmianarayanan_S@infosys.com). method to predict long term stress relaxation. Latte and Rossi [2] showed that on logarithmic plot, duration to leak is a function of inverse absolute temperature and all the points lie on a straight line for a constant pressure. However, these studies are more experimental in nature and do not incorporate the complexities like bolt pre-tension during assemblies. Takashi Kobayashi [3] et al. made a study of the residual bolt force and the sealing performance of flanged connections with ePTFE gaskets based on one year of test. Such studies are complex to set it up in terms of time and cost. It is difficult to optimize the design of leak proof flange using these studies. Using Finite Element Method (FEM) based study, different dimensions of gaskets could be analyzed and the cost of testing can be reduced. So, FEM based study of such complex assembly would be a cost effective approach. However they will be useful only if material model of gaskets are properly captured.

Alkelani et al. [4], proposed a mathematical model of stress relaxation based on Burger model [5], [6] to fit the experimentally evaluated result for styrene butadiene rubber. The same approach is used for the current study, where, the results obtained using burger model is fit for short term (typically < 16Hrs) test data. The aim of the current work is to determine FEM based predictable model for long term (typically $>2$ years) behavior of PTFE. This current approach is used in determining the mathematical model which could be implemented using commercially available FEM software viz. ANSYS. The approximation of gasket is modelled as a Burger model, which is found to be more appropriate for modelling a typical PTFE gasket. ANSYS does not have inbuilt Burger model to accurately predict the unloading behavior of PTFE gaskets. So, the proposed mathematical model is converted in to Prony series which is available in ANSYS. This paper elucidates how the stress relaxation of PTFE gasket behavior can mathematically be modelled and used in ANSYS.

\section{MATHEMATICAL MODELLING OF THE PTFE GASKET}

Stress relaxation is an inherent property of visco-elastic materials. These materials show the behavior of both viscous and elastic materials, which can be modelled by combining elements that represent these characteristics. In this paper Burger model is used for simulating the gasket. Burger's model is basically a Maxwell model [7] in series with the Kelvin-Voigt model [8], [9]. The Fig. 1 shows the proposed model for the gasket.

When gaskets are loaded and released, it partially recovers its original state and this portion of gasket is modelled using an elastic spring of stiffness $E_{m}$. There is a part, that takes considerable amount of time to recover and this time 
dependent recoverable part of gasket is visco-elastic in behavior. It can be modelled using Kelvin-Voigt model constituting of a spring element of stiffness $E_{k}$ and dashpot of $\eta_{k}$ in parallel. Due to compression set a portion of gasket can never bounce back (non-recoverable) is represented by a dashpot of viscosity $\eta_{m}$ that is in series with the Kelvin-Voigt model.

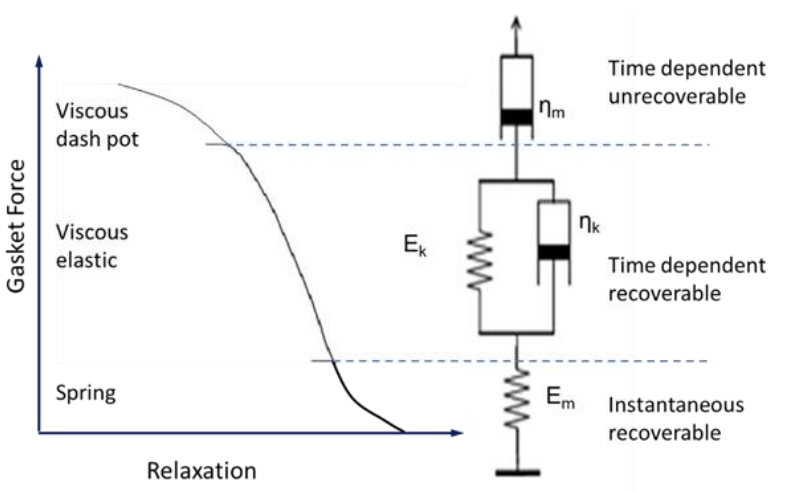

Fig. 1. Mathematical model of gasket.

When a gasket is loaded, its equivalent stiffness reduces with time. This will result in increase of gasket compression $\delta_{g}$ and hence relaxation. Once the bolt is tightened, gasket creep relaxation starts. For a constant compression the force may be expressed as

$$
F=E_{m} \Delta_{1}=\eta_{m} \dot{\Delta}_{2}=\left(E_{k} \Delta_{3}+\eta_{k} \dot{\Delta}_{3}\right)
$$

If a constant spring rate and damping co-efficient are assumed, the deflections $\Delta_{1}, \Delta_{2}$ and $\Delta_{3}$ are as follows,

$$
\begin{gathered}
\Delta_{1}=\frac{F}{E_{m}} \\
\Delta_{2}=\frac{t}{\eta_{m}} F \\
\Delta_{3}=\frac{F}{E_{k}}-\frac{F}{E_{k}} e^{-\left(\frac{E_{k}}{\eta_{k}}\right) t}
\end{gathered}
$$

Thus the total deflection of the gasket $\delta_{g(t)}$ at any given time is obtained by adding (2), (3) and (4)

$$
\delta_{g(t)}=\left[\frac{E_{m}+E_{k}}{E_{m} E_{k}}+\frac{t}{\eta_{m}}-\frac{1}{E_{k}} e^{-\left(\frac{E_{k}}{\eta_{k}}\right) t}\right] F_{(t)}
$$

The stress relaxation experiments as per ASTM F38 [10] are carried out under constant deflection and variation of the gasket load is monitored over a period of time. Hence the above equation can be written as

$$
F_{(t)}=\frac{\delta_{g(t)}}{\left[\frac{E_{m}+E_{k}}{E_{m} E_{k}}+\frac{t}{\eta_{m}}-\frac{1}{E_{k}} e^{-\left(\frac{E_{k}}{\eta_{k}}\right) t}\right]}
$$

The above equation represents the stress relaxation of a gasket over a period of time. By conducting the short duration test and fitting the curve by identifying the constants for experimentally obtained values, the gasket property can be extrapolated for any period of time.

\section{EXPERIMENTAL DATA AND MODEL FITTING}

A creep relaxation curve of an experimental data was obtained from the Fig. 5 of Bergstrom et al. [11]. This curve was further used to obtain the Burger model constants using a curve fitting technique. The Fig. 2 shows that the comparison of experimental data and corresponding burger model after curve fitting. The Table I below shows the required burger model constants obtained from the curve fitting.

TABLE I: BURGER MODEL CONSTANTS

\begin{tabular}{llll}
\hline \hline Constant & Symbol & Value & Unit \\
\hline Maxwell spring stiffness & $E_{m}$ & 4.435 & N/mm \\
Kelvin-Voigt spring stiffness & $E_{k}$ & 12.940 & N/mm \\
$\begin{array}{l}\text { Maxwell dashpot damping coefficient } \\
\begin{array}{l}\text { Kelvin-Voigt dashpot damping } \\
\text { coefficient }\end{array}\end{array} \eta_{m}$ & 20506.158 & N-s/mm \\
\hline \hline
\end{tabular}

It is appropriate to use these constants to develop an analytical material model as we see a good agreement of experimental data and burger model curves $\left(R^{2}=0.993\right)$.

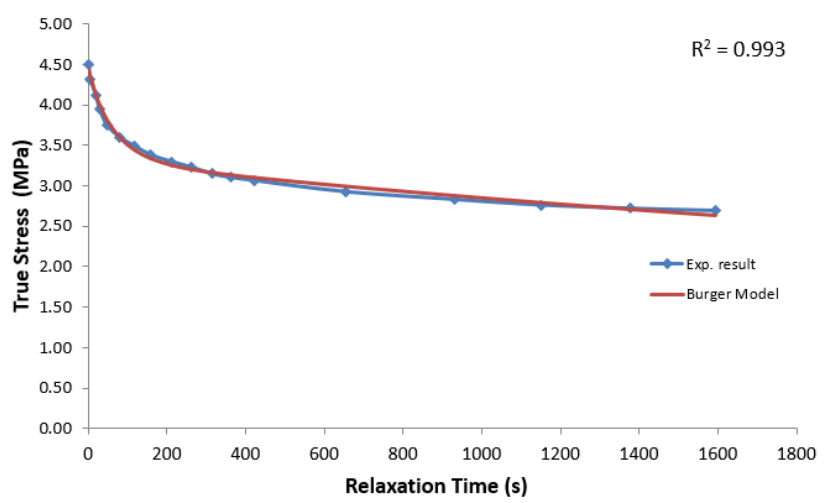

Fig. 2. Relaxation curve for PTFE.

\section{FINITE ELEMENT IMPLEMENTATION}

Commercially available FEM packages like Abaqus, ANSYS do not have a Burger's model implemented in their software. However, they have a generalized visco-elastic model called Prony series, which can be used to model any materials. Using the constants obtained from the experimental data, one can implement the Burger's model in to these FEM based software by tweaking Prony series constants appropriately. The implementation procedure is explained in the section A, where Prony Series is modified to behave like the Burger's model.

\section{A. Conversion of Burger's Model into Prony Series}

Burgers model is a four- element model, defined by the four parameters $\left\{p_{1}, p_{2}, q_{1}, q_{2}\right\}$. We have

$$
\sigma+p_{1} \dot{\sigma}+p_{2} \ddot{\sigma}=q_{1} \dot{\varepsilon}+q_{2} \ddot{\varepsilon}
$$

where the four parameters are expressed in terms of three dimensional shear modulus as 


$$
p_{1}=\frac{\eta_{m}^{\prime} G_{m}+\eta_{m}^{\prime} G_{k}+\eta_{k}^{\prime} G_{m}}{G_{m} G_{k}}, p_{2}=\frac{\eta_{m}^{\prime} \eta_{k}^{\prime}}{G_{m} G_{k}}
$$

And

$$
q_{1}=2 \eta_{m}^{\prime}, q_{2}=2 \frac{\eta_{m}^{\prime} \eta_{k}^{\prime}}{G_{k}}
$$

where,

$$
\begin{gathered}
G_{m}=\frac{E_{m}}{2\left(1+\mu_{m}\right)}, G_{k}=\frac{E_{k}}{2\left(1+\mu_{k}\right)} \text { and } \\
\eta_{m}^{\prime}=\frac{\eta_{m}}{2\left(1+\mu_{m}^{\prime}\right)}, \eta_{k}^{\prime} \frac{\eta_{k}}{2\left(1+\mu_{k}^{\prime}\right)}
\end{gathered}
$$

For viscoelastic material the Poisson's ratios are given by,

$$
\mu_{m}=\mu_{k}=\mu_{m}^{\prime}=\mu_{k}^{\prime}=0.5
$$

Taking Laplace transformation on (7) will yield

$$
\hat{\sigma}+p_{1} s \hat{\sigma}+p_{2} s^{2} \hat{\sigma}=q_{1} \varepsilon_{o}+q_{2} s \varepsilon_{o}
$$

Solving for $\hat{\sigma}$

$$
\hat{\sigma}=\frac{q_{1} s+q_{2} s^{2}}{s\left(1+p_{1} s+p_{2} s^{2}\right)} \varepsilon_{o}
$$

Expanding the above equation by partial fraction and performing the inverse Laplace transformation will yield the stress relaxation,

$$
\sigma_{(t)}=\frac{q_{2}}{p_{2}(\alpha-\beta)}\left[\left(\alpha-\frac{q_{1}}{q_{2}}\right) e^{-\alpha t}+\left(\frac{q_{1}}{q_{2}}-\beta\right) e^{-\beta t}\right]
$$

where: $\alpha, \beta=\frac{p_{1} \pm \sqrt{p_{1}^{2}-4 p_{2}}}{2 p_{2}}$

Expressing (10) in terms of shear relaxation modulus, $G(t)$

$$
G_{(t)}=\frac{G_{m}}{(\alpha-\beta)}\left[\left(\frac{G_{k}}{\eta^{\prime}{ }_{k}}-\beta\right) e^{-\beta t}+\left(\alpha-\frac{G_{k}}{\eta^{\prime}{ }_{k}}\right) e^{-\alpha t}\right]
$$

Prony series [12] consists of a bunch of Maxwell elements in series and adding a spring in parallel with the whole array. The relaxation modulus for this material has the form

$$
G_{(t)}=G_{\infty}+\sum_{i=1}^{N} G_{i} e^{-\left(\frac{t}{\tau_{i}}\right)}
$$

where, $G_{\infty}$ is the steady-state stiffness (represented by the parallel spring), and $G_{\mathrm{i}}, \tau_{i} i=1 \ldots N$ are the time constants and represented by stiffness's of the Max well elements. These parameters are used directly as the properties of the material. A two parameter expansion would look like

$$
G_{(t)}=G_{\infty}+G_{1} e^{-\left(\frac{t}{\tau_{1}}\right)}+G_{2} e^{-\left(\frac{t}{\tau_{2}}\right)}
$$

Comparing the above two equations (13) and (11), we have,

$$
\begin{gathered}
G_{\infty}=0, G_{1}=\frac{G_{m}}{(\alpha-\beta)}\left(\frac{G_{k}}{\eta_{k}^{\prime}}-\beta\right), \\
G_{2}=\frac{G_{m}}{(\alpha-\beta)}\left(\alpha-\frac{G_{k}}{\eta_{k}^{\prime}}\right), \tau_{1}=\frac{1}{\beta} \text { and } \tau_{2}=\frac{1}{\alpha}
\end{gathered}
$$

Since ANSYS uses relative moduli [13] i.e.

$$
g_{(t)}=\frac{G_{(t)}}{G_{m}}
$$

Hence the material constants that needs to be used in ANSYS are

$$
\begin{gathered}
g_{1}=\frac{1}{(\alpha-\beta)}\left(\frac{G_{k}}{\eta_{k}^{\prime}}-\beta\right), g_{2}=\frac{1}{(\alpha-\beta)}\left(\alpha-\frac{G_{k}}{\eta_{k}^{\prime}}\right), \\
\tau_{1}=\frac{1}{\beta} \text { and } \tau_{2}=\frac{1}{\alpha}
\end{gathered}
$$

The above material parameters can be suitably used in ANSYS or any other FE packages to represent the burger material model.

For example, the commands to implement the material model in ANSYS would look like

\section{TB, PRONY, 1, 1, 2, SHEAR}

\section{TBTEMP, 0}

TBDATA, $g 1, \tau 1, g 2, \tau 2$,

The above mathematical model works with the assumption that the gasket stiffness remains constant for different stress levels.

\section{B. Validation of Burger's Model Using ANSYS}

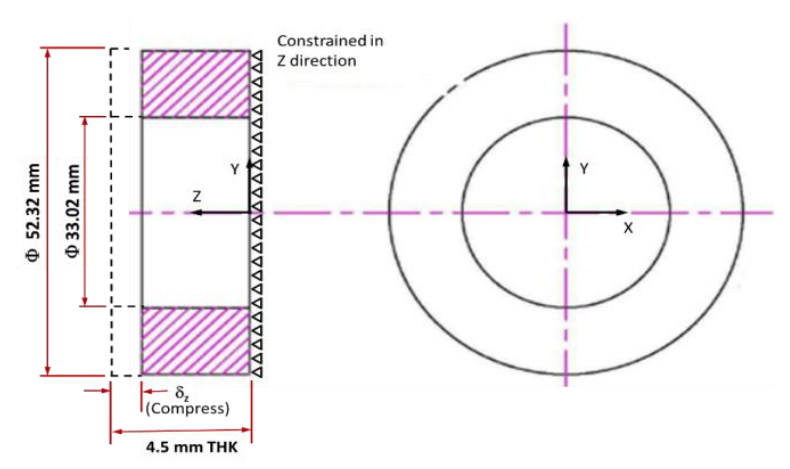

Fig. 3. FEM Modeling of PTFE Gasket.

A finite element model was developed to verify the behavior of PTFE material. The model is an annular specimen of ID $33.02 \mathrm{~mm}$ and OD of $52.32 \mathrm{~mm}$ with a thickness of $4.5 \mathrm{~mm}$ as shown in the Fig. 3. The model was meshed with higher order 3-D 20-node solid element (SOLID186). These elements exhibit quadratic displacement behavior with three degrees of freedom per node. Bottom side of the specimen is constrained in the axial direction. The top side of the specimen was given displacement boundary condition such that the model is under a uniform stress of 4.5MPa for the first load step. The same displacement has been maintained over a period of 26 minutes $(1600 \mathrm{sec})$ with creep rate option "on". Then the axial stress was verified for the first load step to check if the specimen is induced with the 
required stress. The Fig. 4 shows that the stress at the end of first load step is $4.5 \mathrm{MPa}$.

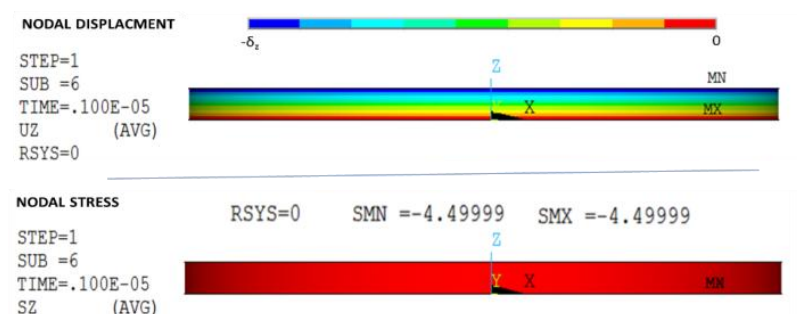

Fig. 4. Compressive Stress at the end of Initial loading.

The stress relaxation behavior was captured for corresponding transient time period $(1600 \mathrm{sec})$ and the same is plotted in the Fig. 5. The time history stress values obtained from the ANSYS are plotted as relaxation curve and the same is compared with the experimental results.

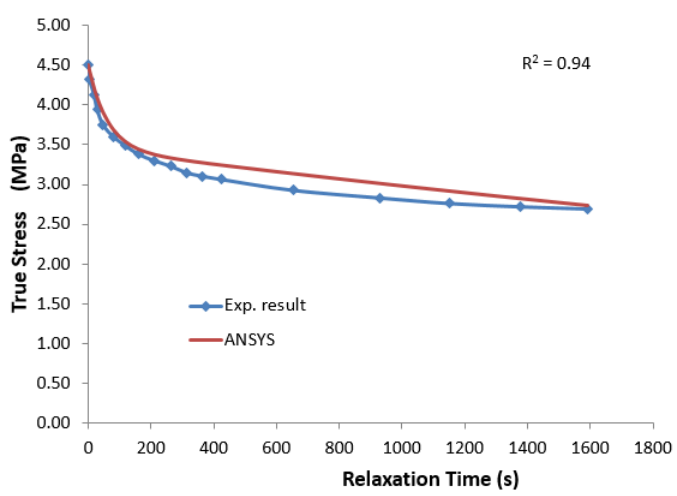

Fig. 5. Experimental and FEM predicted relaxation result.

From Fig. 5, it can be concluded that there is a good agreement between the experimental data to that predicted by ANSYS, with a $R^{2}$ value of 0.94 . Hence, this material model can be used for any components made up of PTFE in the FEA software.

\section{CONCLUSION}

The detailed study on how to develop and implement a material model for PTFE is discussed in this paper. The comparative result of experimentally measured data and analytically fit data demonstrate a high precision of the Burgers model for prediction of the stress relaxation curve. Thus, the model can describe the overall time-dependent behavior of PTFE gaskets.

Four element Burgers model can be represented using a generalized model like prony series and can be used directly in the commercially available FEA software like ANSYS and Abaqus. The comparative result of experimentally measured data and results obtained from ANSYS demonstrate a good agreement.

This methodology can be adopted in finite element analysis models to obtain valuable information on stresses distribution in the gasket and on stress relaxation during the functioning of the seal, allowing this way to define the optimal maintenance procedures.

\section{REFERENCES}

[1] D. L. Merchand and Michel, "Long term performance of elastomeric sheet gasket materials subjected to temperature exposure," in Proc. the $19968^{\text {th }}$ International Conference on Pressure Vessel Technology, 1996, pp. 107-123.
[2] L. Jorg and R. Claudi, "High-temperature behavior of composite fiber material and an alternative approach of life expectancy statement," in Proc. Fifth Annual Technical Symposium of the Fluid Sealing Association, 1994, pp. 171-186.

[3] T. Kobayashi, T. Hagiri, K. Nishiura, M. Hiratsuka, and Katsutoyo, "The residual bolt force and the sealing performance of flanged connections with expanded PTFE gaskets," in Proc. ASME2012 Pressure Vessels and Piping Conference, Ontario, Canada.

[4] A. A. Alkelani, B. A. Housari, and S. A. Nassar, "A proposed model for creep relaxation of soft gaskets in bolted joints at room temperature," Journal of Pressure Vessel Technology, vol. 130, pp. 1-6, 2008.

[5] D. E. Czernik, Gaskets Design, Selection, and Testing, McGraw-Hill, New York, 1996, pp. 51-54, 89-92.

[6] G. W. Ehrenstein, Polymeric Materials: Structure Properties Applications, Hanser, Munich, 2001.

[7] J. C. Maxwell, "On the dynamic theory of gases," Philosophical Transactions of the Royal Society of London, vol. 157, pp. 49-88, 1867.

[8] L. Kelvin, "On the elasticity and viscosity of metals," Proceedings of the Royal Society, vol. 14, pp. 289-297, 1865.

[9] W. Voigt, "Ueber innere Reibung fester K"orper, insbesondere der Metalle," Annalen der Physik, vol. 283, no. 12, pp. 671-693, 1892.

[10] F38-00, ASTM. Standard Test Methods for Creep Relaxation of a Gasket Material.

[11] J. S. Bergstrom and L. B. Hilbert, "A constitutive model for predicting the large deformation, thermomechanical behavior of fluoropolymers," Mechanics of Materials, vol. 37, issue 8, pp. 899-913, August 2005.

[12] ANSYS 15 user guide, section 3.7, Viscoelasticity.

[13] ANSYS 15 user guide, section 4.9, Viscoelasticity.

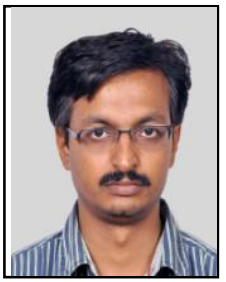

Madhu Bharadwaj was born in Bangalore, India in 1979. He received the bachelor of engineering (B.E.) degree in mechanical engineering from the Bangalore University, India in 2000 and the master of engineering (M.E) in production technology from Madurai University in 2003. He has master of science (M.Sc.) in total quality management from Sikkim Manipal University of Health, Medical and Technological Sciences, India. Since September 2012 he has been working for engineering services group of Infosys Limited. He is currently a lead consultant of Advanced Engineering Group and engaged in execution and solving technical problems for various turbo machinery customers. Prior to Infosys, he worked for general electric where he has extensively worked in the design of turbo-generator. He has been involved with several new product development projects at GE. He also has three patents (US 2009/0260221 A1, US 20120098385 and US 8998588 B2) in his credential.

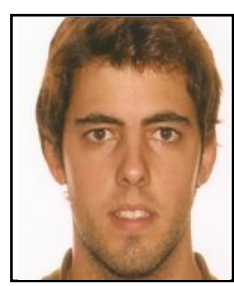

Santiago Claramunt Estecha was born in Catalunya, Spain, in 1985. He received the master of science (M.Sc.) degree in mechanical engineering from universitat Politècnica de Catalunya, Spain, in 2008. He is currently working as a PMSG (Permanent magnet synchronous generator) mechanical technical leader at Alstom Power since 2008. He has been involved with several new product development projects at Alstom Wind. He also has couple of patents in his credential

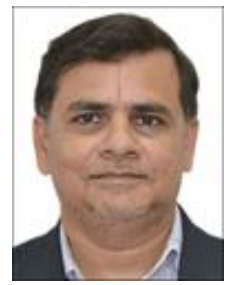

Sowmianarayanan Srinivasan was born in Kalayarkoil a small village in India, in 1969. He received the B.E. degree in mechanical engineering from the College of Engineering, Anna University of Chennai, India, in 1997, and the Master of Technology in computational engineering from the Indian Institute of Technology Madras (IITM), Chennai, India, in 2005

Since January 2009, he has been working for engineering services group of Infosys Limited. He is currently a principal consultant of Advanced Engineering Group and engaged in execution and mentoring of solving technical problems for various gas turbine engine customers. Prior to Infosys, he was specialized in solving non-linear material, large deformation and contact problems of various mechanical systems using finite element analysis. He has worked in various domains, which includes turbo machinery, medical devices and heavy machineries. He has authored one international journal paper and presented number of national and international conferences. He was the recipient of best paper award during ANSYS conference during 2014 held in India. 